Network Working Group

Request for Comments: 1954

Category: Informational
P. Newman, Ipsilon

W. L. Edwards, Sprint

R. Hinden, Ipsilon

E. Hoffman, Ipsilon

F. Ching Liaw, Ipsilon

T. Lyon, Ipsilon

G. Minshall, Ipsilon May 1996

\title{
Transmission of Flow Labelled IPv4 on ATM Data Links Ipsilon Version 1.0
}

Status of this Memo

This document provides information for the Internet community. This memo does not specify an Internet standard of any kind. Distribution of this memo is unlimited.

\section{IESG Note:}

This note documents a private protocol for IPv4-based flows. This protocol is NOT the product of an IETF working group nor is it a standards track document. It has not necessarily benefited from the widespread and in depth community review that standards track documents receive.

\section{Abstract}

This document specifies the manner for transmitting IPv4 datagrams over an ATM data link, both in a default manner and in the presence of flow labelling via Ipsilon Flow Management Protocol [IFMP].

Table of Contents

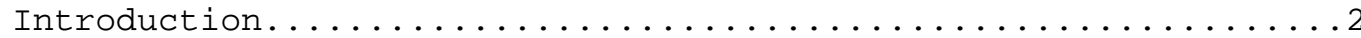

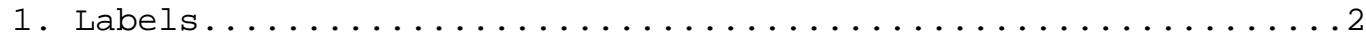

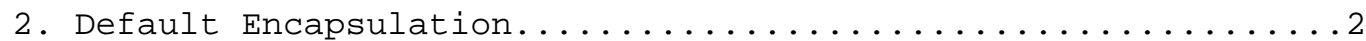

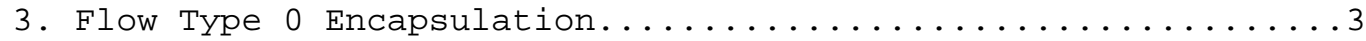

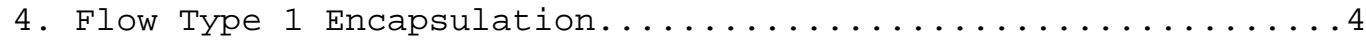

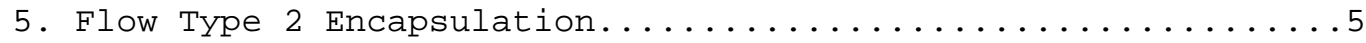

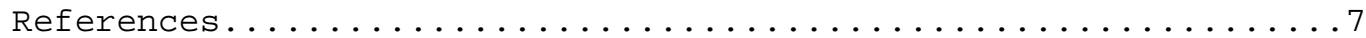

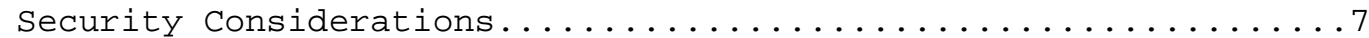

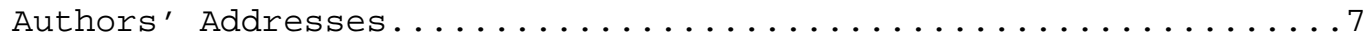




\section{Introduction}

This document specifies the manner for transmitting IPv4 datagrams over an ATM data link, both in a default manner and in the presence of flow labelling via Ipsilon Flow Management Protocol [IFMP]. ATM specific functions such as OAM cells, the CLP bit, and ABR RM cells are not used. There are no reserved VCIs other than VPI = 0 , VCI = 0 , which indicates an unassigned cell; and VPI = 0, VCI = 15, which is used for the default encapsulation. IFMP messages must be sent using the default encapsulation.

1. Labels

Labels, as carried by IFMP, are realized on an ATM data link as specific VPI/VCIs. The format of the Label field for ATM labels is:

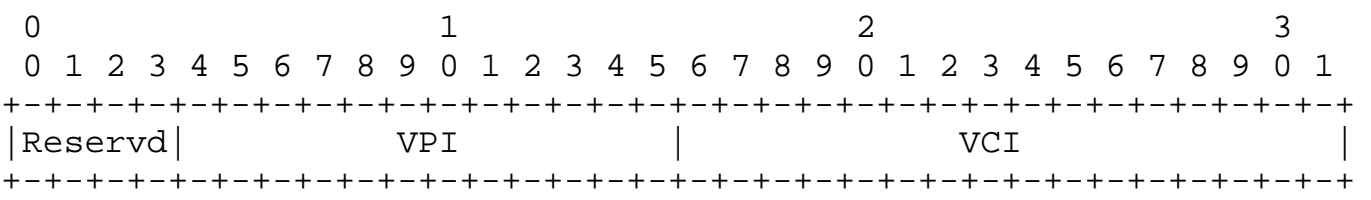

The low order 16 bits of the label correspond to the VCI, with the least significant bit of the Label field corresponding to the least significant bit of the VCI. If the link cannot support a full 16 bit VCI the unused bits of the VCI must be the most significant bits and they must be set to zero.

The next 12 higher order bits of the label correspond to the VPI, with the least significant of these bits corresponding to the least significant bit of the VPI. If the link cannot support a full 12 bit VPI, then the unused bits of the VPI must be the most significant bits and they must be set to zero. The most significant four bits of the label are reserved. They should be set to zero by the sender and ignored by the receiver.

\section{Default Encapsulation}

The default encapsulation for IPv4 packets on ATM data links is the LLC/SNAP encapsulation specified in section 4.1 "LLC encapsulation for routed protocols" of RFC 1483 [RFC1483]. Such frames begin with the octets OxAA 0xAA 0x03 0x00 0x00 0x00 0x08 0x00 (the LLC/SNAP header for IPV4). The LLC/SNAP header is prefixed to the IP datagram and the entire packet is encapsulated within the payload of an AAL-5 CPCS-PDU as specified in RFC 1483 and illustrated below: 


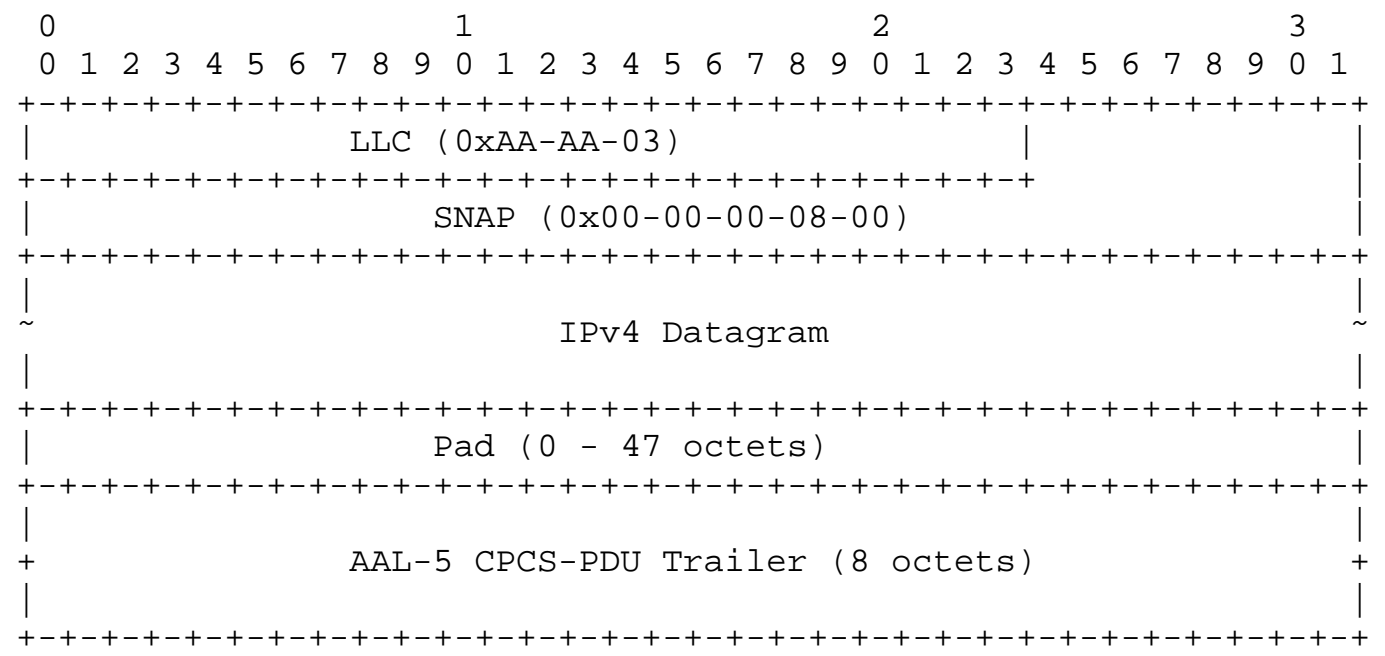

The maximum transmission unit (MTU) of an IPv4 datagram using the default encapsulation is 1500 octets.

Frames using the default encapsulation are sent to: VPI $=0$, VCI = 15 .

3. Flow Type 0 Encapsulation

All IPv4 frames using Flow Type 0 are encapsulated within the payload of an AAL-5 CPCS-PDU. This is the null encapsulation of section 5.1 "VC based multiplexing of routed protocols" from RFC 1483 [RFC1483]. There is no LLC/SNAP header. The first octet of the frame corresponds to the first octet of the IPv4 datagram (i.e., the octet that contains the IP version number (4) and Internet Header Length (IHL) ). The IP datagram is encapsulated within the payload of an AAL-5 CPCS-PDU as specified in RFC 1483 and illustrated below: 


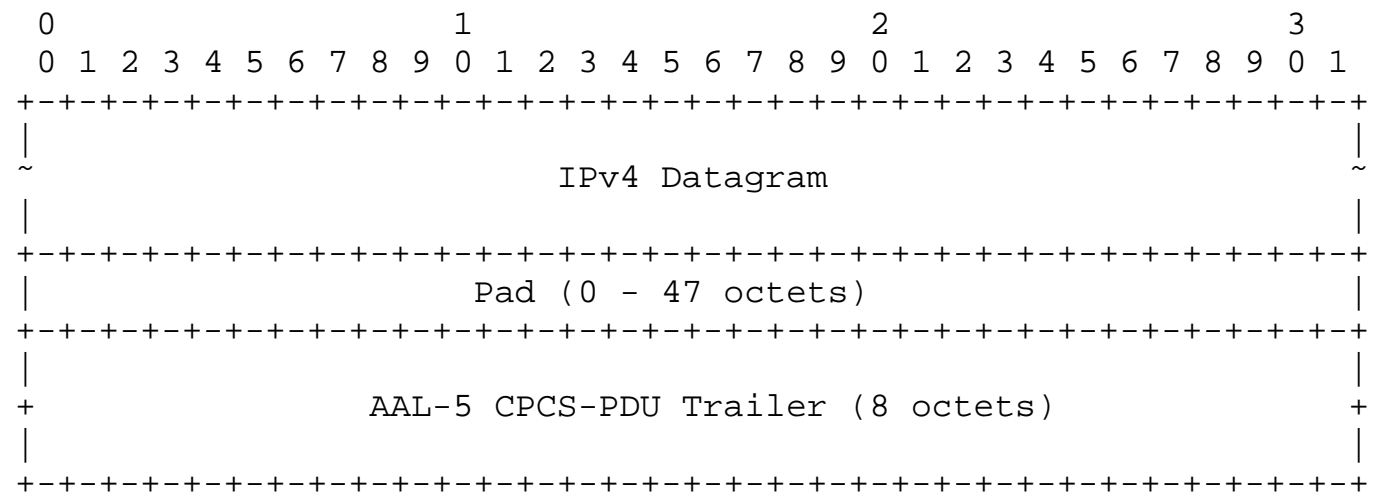

The MTU of an IPv4 datagram using Flow Type 0 encapsulation is 1500 octets.

Frames using Flow Type 0 encapsulation are sent to the VPI/VCI specified in the Label field of the corresponding IFMP Flow Type 0 Redirect message element [IFMP].

4. Flow Type 1 Encapsulation

All IPv4 frames using Flow Type 1 are encapsulated directly in the payload of an AAL-5 CPCS-PDU. This is the null encapsulation of section 5.1 "VC based multiplexing of routed protocols" from RFC 1483 [RFC1483]. There is no LLC/SNAP header. Also, the following fields of the IP header are not transmitted: Version, Internet Header Length (IHL), Type of Service (TOS), Time to Live (TTL), Protocol, Source Address, and Destination Address. In addition, the first 4 octets immediately following the IP header (as determined by the IHL field) are not transmitted. (These 4 octets correspond to the source and destination ports for TCP and UDP datagrams.) The value of the Total Length field is not changed; it remains the total length of the IP datagram before the above fields were removed. The transmitted value of the Checksum field is the checksum value that would have been computed for the entire IP header if the TTL field had been set to zero (i.e., the actual value of the TTL field is "subtracted", using one's-complement arithmetic, from the Checksum before transmission).

The IP datagram is encapsulated within the payload of an AAL-5 CPCSPDU as specified in RFC 1483 and illustrated below: 


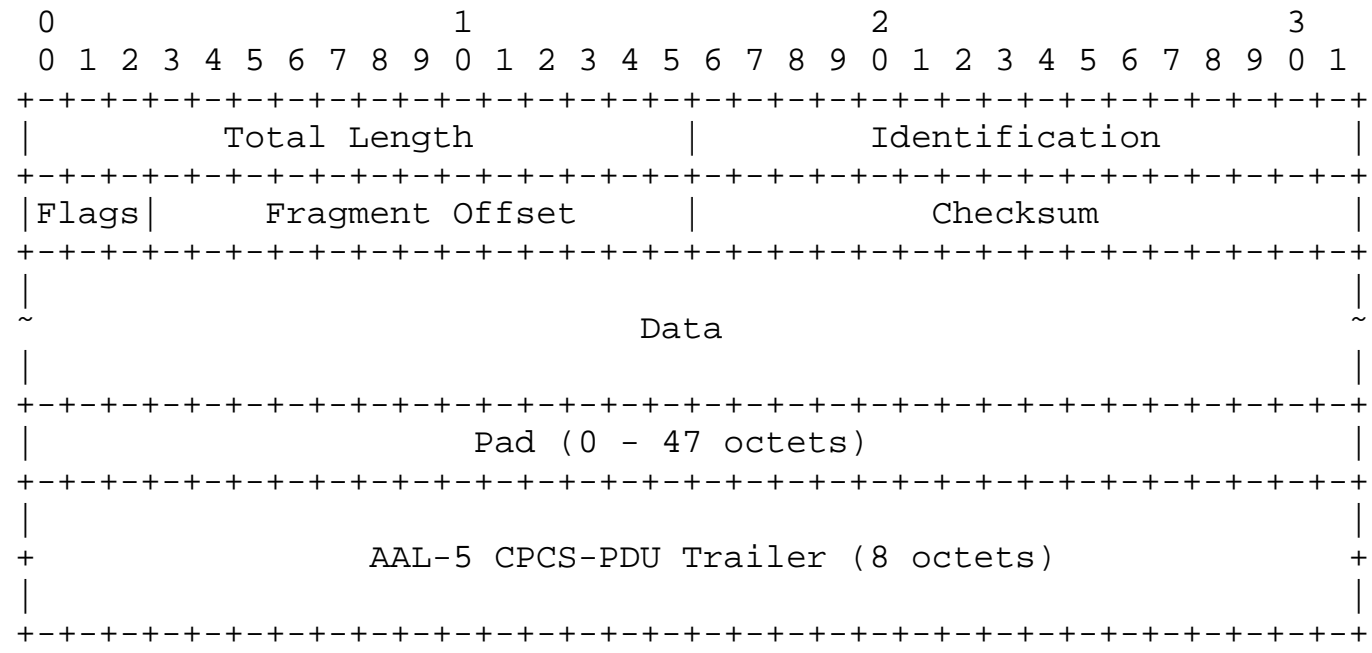

The MTU of the IPv4 datagram using Flow Type 1 encapsulation is 1484 octets (1500 octets minus the 16 octets specified above).

Frames using Flow Type 1 encapsulation are sent to the VPI/VCI specified in the Label field of the corresponding IFMP Flow Type 1 redirect message element [IFMP].

5. Flow Type 2 Encapsulation

All IPv4 frames using Flow Type 2 are encapsulated directly in the payload of an AAL-5 CPCS-PDU. This is the null encapsulation of section 5.1 "VC based multiplexing of routed protocols" from RFC 1483 [RFC1483]. There is no LLC/SNAP header. Also, the following fields of the IP header are not transmitted: Version, Internet Header Length (IHL), Time to Live (TTL), Source Address, and Destination Address. The first 4 octets immediately following the IP header (as determined by the IHL field) are transmitted. (These 4 octets correspond to the source and destination ports for TCP and UDP datagrams.) The value of the Total Length field is not changed; it remains the total length of the IP datagram before the above fields were removed. The transmitted value of the Checksum field is the checksum value that would have been computed for the entire IP header if the TTL field had been set to zero (i.e., the actual value of the TTL field is "subtracted", using one's-complement arithmetic, from the Checksum before transmission).

The IP datagram is encapsulated within the payload of an AAL-5 CPCSPDU as specified in RFC 1483 and illustrated below: 


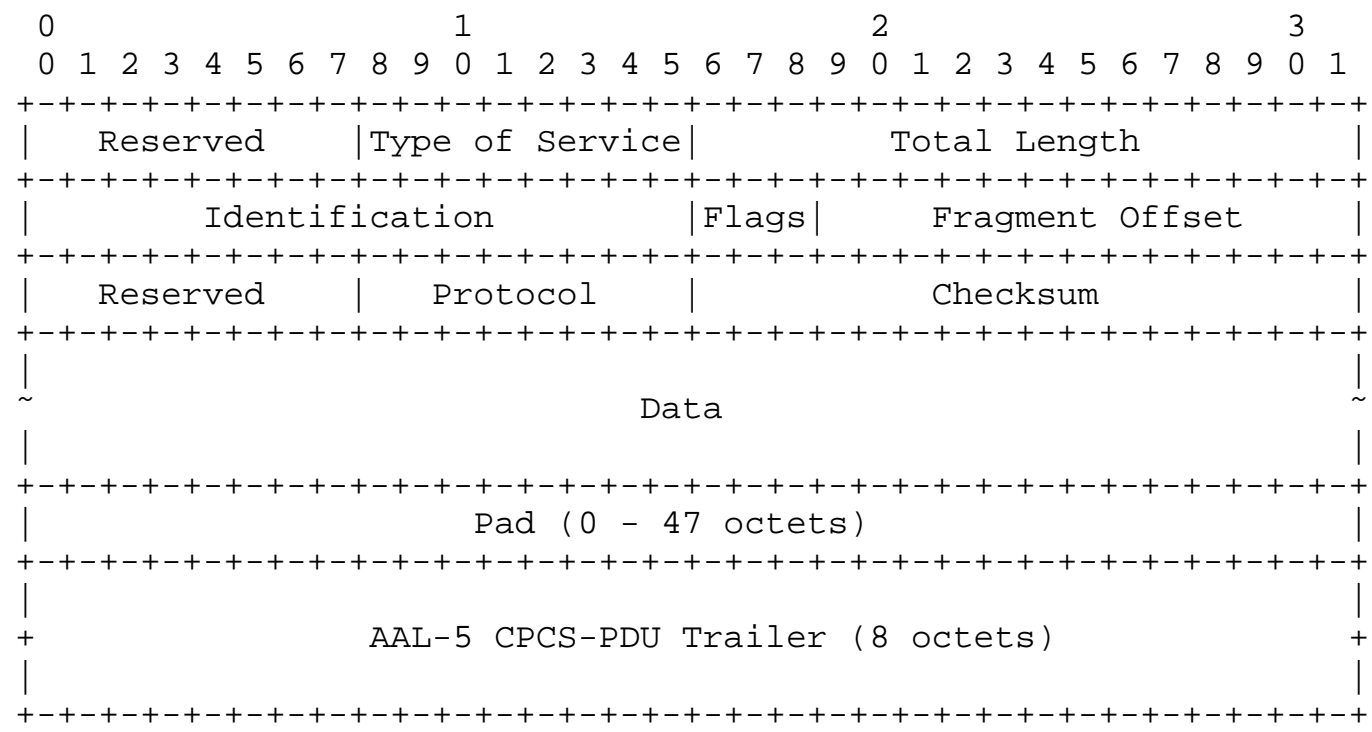

The Reserved fields are not used and should be set to zero by the sender and ignored by the receiver.

The MTU of the IPv4 datagram using Flow Type 2 encapsulation is 1492 octets (1500 octets minus the 8 octets specified above).

Frames using Flow Type 2 encapsulation are sent to the VPI/VCI specified in the Label field of the corresponding IFMP Flow Type 2 redirect message element [IFMP]. 
References

[IFMP] Newman, P., et. al., "Ipsilon Flow Management Protocol Specification for IPV Version 1.0", Ipsilon Networks Inc., RFC 1953, May 1996.

[RFC1483] Heinanen, J., "Multiprotocol Encapsulation over ATM Adaptation Layer 5", RFC 1483, July 1993.

SECURITY CONSIDERATIONS

Security issues are not discussed in this document.

AUTHORS' ADDRESSES

Peter Newman

Ipsilon Networks, Inc.

W. L. Edwards, Chief Scientist

Sprint

Robert M. Hinden

Ipsilon Networks, Inc.

Eric Hoffman

Ipsilon Networks, Inc.

Fong Ching Liaw

Ipsilon Networks, Inc.

Tom Lyon

Ipsilon Networks, Inc.

Greg Minshall

Ipsilon Networks, Inc.

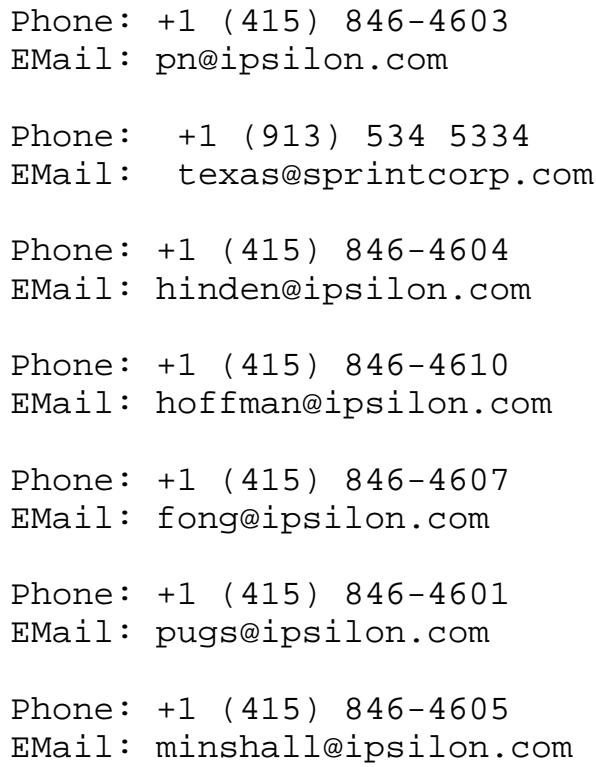


Ipsilon Networks, Inc. is located at:

2191 East Bayshore Road

Suite 100

Palo Alto, CA 94303

USA

Sprint is located at:

Sprint

Sprint Technology Services - Long Distance Division

9300 Metcalf Avenue

Mailstop KSOPKB0802

Overland Park, KS 66212-6333

USA 